\title{
Révolution constituante et société judiciaire
}

L'exemple septentrional

The Revolution of the Constituent Assembly and Judicial Society: the Northern example

Hervé Leuwers

\section{CpenEdition}

Journals

Édition électronique

URL : https://journals.openedition.org/ahrf/11214

DOI : 10.4000/ahrf.11214

ISSN : 1952-403X

Éditeur :

Armand Colin, Société des études robespierristes

Édition imprimée

Date de publication : 1 décembre 2007

Pagination : 27-47

ISSN : 0003-4436

Référence électronique

Hervé Leuwers, "Révolution constituante et société judiciaire », Annales historiques de la Révolution française [En ligne], 350 | octobre-décembre 2007, mis en ligne le 01 janvier 2011, consulté le 21 septembre 2021. URL : http://journals.openedition.org/ahrf/11214; DOl : https://doi.org/10.4000/ ahrf. 11214 


\title{
RÉVOLUTION CONSTITUANTE ET SOCIÉTÉ JUDICIAIRE L'EXEMPLE SEPTENTRIONAL
}

Hervé LEUWERS

\begin{abstract}
De 1789 à 1791, par la réorganisation des tribunaux, la suppression de la vénalité des charges, l'abolition des anciens corps de professionnels du droit ou la redéfinition de la représentation et de la défense en justice, l'Assemblée constituante modifie en profondeur le groupe des gens de loi. L'examen de l'application des réformes, particulièrement à partir des exemples du Nord et du Pas-de-Calais, permet la mise en évidence de leurs implications géographiques, professionnelles et sociales. Derrière l'importance du bouleversement d'ensemble, tantôt voulu par les députés, tantôt implicite, ce sont des effets contrastés - voire contradictoires - qui apparaissent : le renouvellement géographiquement et professionnellement variable des personnels, leur resserrement numérique, leur spécialisation fonctionnelle, leur définition nationale, leurs mutations culturelles... C'est l'ampleur du changement et, plus encore, l'adaptation subie ou assumée de nombreux gens de loi d'Ancien Régime à une nouvelle justice qui se donne à voir.
\end{abstract}

Mots-clés : juge, avocat, procureur, greffier, huissier, Nord, Pas-de-Calais.

Des réformes entreprises par l'Assemblée constituante, celle de la justice apparaît comme l'une des plus ambitieuses et des plus abouties ; il est vrai que l'ampleur et la variété de ses enjeux en font l'une des clefs de l'ordre nouveau. Comment, en effet, concevoir l'État régénéré sans une justice nationalisée, rationalisée et profondément transformée dans sa résolution des différends et sa répression du crime ? À l'éclatement juridictionnel entre tribunaux seigneuriaux, municipaux et royaux se substitue une 
structure cohérente et unifiée ; à la complexité des anciens ressorts succède un cadre rationnel, car inscrit dans le nouveau maillage administratif ; mais surtout, le roi de justice cède le pas à la Nation, qui gagne le droit d'élire ses juges et de participer à la justice criminelle par l'intermédiaire du double jury, tandis que les lois pénales sont réformées et codifiées. Au-delà des enjeux politiques, la réforme permet l'établissement d'une justice plus efficace et humaine au pénal, plus rapide et moins onéreuse au civil - même si, en ce dernier domaine, l'unité du droit et le complet renouveau des procédures sont reportés à plus tard -. Au fur et à mesure de la mise en place des tribunaux nouveaux, de 1790 au printemps 1792 , le fonctionnement de la justice prend un nouveau visage. Au civil, priorité est donnée à l'arbitrage et à la conciliation devant des arbitres, un tribunal de famille ou le bureau de paix, voire le seul juge de paix, l'accès aux juges du tribunal de district ne devant intervenir qu'en dernier recours et avec un seul appel possible à l'issue du jugement ; au pénal, selon les cas, c'est le tribunal de police municipale, celui de police correctionnelle, le tribunal de district en appel des précédents - ou le tribunal criminel de département qui juge. $\mathrm{Au}$ sommet de l'édifice judiciaire, le Tribunal de cassation trône comme juge suprême du respect du droit et des procédures'.

Au-delà de ses enjeux politiques et judiciaires, la réforme peut être vécue, particulièrement par les gens de justice, comme une rupture professionnelle et sociale majeure ${ }^{2}$. Le processus enclenché, cependant, n'est pas sans contradictions. D'un côté, c'est la disparition ou la transformation de certaines fonctions, une diminution drastique du nombre des gens de justice, leur nouvelle répartition sur le territoire, l'abolition des privilèges traditionnellement reconnus aux juges ou avocats qui obligent les juristes en place à renoncer à leurs statuts, parfois à s'adapter professionnellement dans un cadre géographique qui n'est pas toujours celui de leurs anciennes fonctions. Mais de l'autre, ce sont des processus à l'œuvre dès l'Ancien Régime qui, par la volonté de l'Assemblée ou implicitement, prennent un nouvel essor, comme le resserrement professionnel de certaines activités ou la construction nationale des groupes professionnels. Pour restituer ces enjeux et ces transformations, examiner l'adaptation souvent difficile des gens de loi à l'ordre nouveau, il faut délaisser l'analyse des discours politiques, des structures judiciaires ou de l'engagement politique des juristes ; c'est par l'observation locale, ici d'abord menée dans les départements du Nord et du Pas-de-Calais, qu'ils transparaissent.

(1) Sur la réforme judiciaire et ses suites, voir : Jean-Pierre RoYf.r. Histoire de la justice en France, Paris, PUF, $2^{c}$ éd. 1996, p. 235-330; Jean-Claude FARCY, $L$ h'histoire de la justice française de la Révolution à nos jours, Paris, PUF, 2001, p. 175-193; Philippe Boucher, dir., La Révolution et la justice. Des lois du roi au droit modeme, Paris. J.-P. de Monza, 1989.

(2) Une approche sociale de la réforme est présente dans Isser Woloch, The New Regime. Transformations of the French Civic Order, 1789-1820 s., New York \& London, W.W. Norton \& Company, 1994, p. 321-326 et Jean-Pierre ROYER, Histoire de la justice..., op. cit., p. 302-321. 


\section{Le temps d'un renouveau professionnel}

En annonçant la disparition des juridictions établies, la remise à plat de la carte judiciaire, la suppression de la vénalité et la transformation des professions juridiques, l'Assemblée constituante ouvre une période d'incertitudes qui ne s'interrompt qu'après l'installation des tribunaux criminels, au printemps $1792 . .$. pour reprendre dès les lendemains du 10 Août. Entre temps, en l'espace de deux années, les fonctions et les statuts des professionnels de la justice sont redéfinis, et les nouvelles places distribuées ; pour des députés souhaitant établir un accès rationalisé et limité à la justice civile ordinaire, celle rendue par les tribunaux de district, la réforme passe par une diminution du nombre des professionnels du droit à laquelle les intéressés réagissent diversement.

Dès 1790 , les juges savent qu'ils devront céder leur place à d'autres ; la suppression des justices municipales et seigneuriales, de même que la fín de la vénalité des offices royaux annoncent une importante redistribution des rôles. En décidant que les juges nouveaux seraient élus (mai 1790), l'Assembléc répond à un vœu fréquemment formulé par les cahiers de doléances ${ }^{4}$, notamment dans les provinces du Nord ${ }^{5}$. Face à ces mesures, les magistrats parlementaires ou présidiaux et, à plus forte raison ceux des juridictions inférieures, n'expriment aucune protestation d'ensemble ; à peine peut-on relever, à Paris, Metz ou Rouen, quelques regrets formulés à l'occasion de la prolongation des vacances parlementaires (3 novembre 1789), ou lors des dernières séances des juridictions supprimées'. Dans l'espace septentrional, comme en 1788 , la transition s'effectuc sans incident connu, les greffiers, huissiers-audienciers et sergents se soumettant, comme les juges, au grand renouvellement de personnels des tribunaux de l'automne 1790 .

Dans ce vaste renouveau des professions juridiques, l'histoire des procureurs fait exception. Certes, ils savent dès 1789 que leurs offices vont disparaître, et qu'un texte viendra réglementer leurs nouvelles fonctions ; mais, le temps aidant, ils s'organisent et mènent une vaste campagne de protestation auprès des autorités, dans laquelle ils défendent l'utilité de

(3) Décrets des 5 et 7 mai $1790 \mathrm{ct}$ loi des 16 et 24 août 1790. Voir Jean-Pierre RoYER, Histoire de la justice..., op. cit., p. $311-319$.

(4) Même si, sur ce point, des propositions diverses furent faites au printemps 1789. Voir Arlette Lebigre, " 1789 : la justice dans tous ses états ", dans Robert BAdinter, dir., Une autre justice, Paris, Fayard, 1989, notamment p. 45-46.

(5) Voir les cahiers de doléances de la ville de Saint-Omer, du Tiers état du bailliage de Calais et du clergé du bailliage de Montreuil, dans Jean-Pierre Jessennf et Dominique RosselLe, Florilege des cahiers de doléantces du Pas-de-Calais, Lille, CHRN, 1989, p. 165, 204 et 250.

(6) Edmond Seligman. La justice en France pendant la Révolution (1789-1792), Paris, Plon-Nourrit, 1901, p. 221-226. 
leur fonction, revendiquent son maintien et, au besoin, la réduction du nombre de leurs charges. Dès le printemps 1790, le mouvement est lancé par les communautés de Poitiers, Marseille, Aix ou le Puy, qui écrivent à leurs confrères du Châtelet de Paris ; dans les mois qui suivent, le mouvement gagne en ampleur et de nombreux mémoires parviennent aux Comités de Judicature et de Constitution?. Entre décembre 1790 et janvier 1791, lorsque la question est à l'ordre du jour de l'Assemblée, des députés comme Dinocheau ou Tronchet parviennent à faire confier le suivi des procédures à un professionnel du droit, également apte à plaider, que l'on appelle l'avoué (décret des 29 janvier-20 mars 1791); il sera le successeur désigné du procureur, tandis que le défenseur officieux sera celui de l'avocat.

C'est au détour d'un décret complémentaire sur l'organisation judiciaire (2-11 septembre 1790), par l'évocation du costume professionnel des gens de justice, que la Constituante scelle la suppression des ordres et l'abandon du mot " avocat ". Les " hommes de loi ", bientôt désignés comme " défenseurs officieux ", perdent leur quasi monopole de la défense, puisque les avoués y ont un accès plus large que les anciens procureurs et que le droit de tout un chacun à défendre sa propre cause, tant au civil qu'au pénal, est reconnu par l'Assembléc depuis le décret des 16-24 août 1790. Face à ces changements, le silence des ordres est pratiquement général ; il est vrai que, parfois dès 1789 , ils ont cessé de se réunir et n'ont plus renouvclé leurs responsables, la loi nouvelle les incitant souvent à liquider leurs communautés. Dans un premier temps, d'ailleurs, ils peuvent se survivre à eux-mêmes en apparaissant comme " hommes de loi " dans les almanachs et devant les tribunaux*.

Labsence de réaction aux transformations des statuts des gens de loi, dans le Nord et le Pas-de-Calais, ne signifie pas adhésion inconditionnelle aux lois nouvelles; elle peut aussi trahir, aux débuts de la réforme, la certitude d'une poursuite possible d'une activité, l'espoir d'un reclassement ou la résignation, notamment pour ces nombreux avocats en titre qui, en 1790, perdent plus un statut qu'une profession. C'est lorsque les espoirs sont contrariés que les sources révèlent des réactions multiples ; elles mettent alors en évidence la variété géographique des situations et l'ampleur différenciée des changements annoncés.

(7) Par cxemple, AN, D IV 20, dos. 412, pièce 5 (Aix) : D IV 21, dos. 434, pièce 20 (Vicomté de Saint-Pierre) : D IV 25, dos. 584, pièce 11 (Bourganeuf)... Voir aussi : Edmond SElugan, op. cii., p. $311-318$.

(8) Hervé LEUWERs, L'invention du barreau français, 1660-1830. La consinuction nationule d'un groupe professionnel, Paris, Ed. EHESS, 2006, p. 243-250. 


\section{Enjeu social et espace de la réforme}

D'une ville à l'autre, l'impact social de la réforme varie en fonction de l'importance de la vie judiciaire d'Ancien Régime et de la place obtenue dans la nouvelle hiérarchie des tribunaux. Au septentrion, les départements du Nord et du Pas-de-Calais sont l'un et l'autre distribués en huit districts, qui constituent les ressorts d'autant de tribunaux composés chacun - sauf exception - de cinq juges et de quatre suppléants élus, d'un commissaire du roi nommé à vie par le monarque et d'un greffier désigné par les juges"; au printemps 1792, un tribunal criminel vient compléter le dispositif dans chacun des départements. Dans un espace étroitement encadré par les justices royales, seigneuriales ou municipales d'Ancien Régime, cette réforme, de même que le transfert d'une partie du contentieux à de modestes juges de paix ou à des instances arbitrales ou conciliatoires, remet en cause les fonctions judiciaires de bien des communes; outre l'enjeu politique, qui conduit les villes à s'engager pour l'obtention d'un tribunal - par un jeu de pressions ou d'interventions auprès des députés ou des comités de l'Assemblée -, qui rappelle les débats et les combats pour l'obtention d'un chef-lieu de département ou de district ${ }^{10}$, la redistribution des rôles oblige nombre d'anciens magistrats, avocats, procureurs, greffiers ou huissiers à s'interroger sur leur avenir professionnel. Pour les uns, l'ordre nouveau est une promesse d'activité, parfois plus dense qu'autrefois, alors que d'autres voient leur ville privée d'un véritable tribunal. Par son choix de n'établir que 545 tribunaux de district en province, le décret du 23 août 1790 annonce des implications sociales contrastées", que les départements les plus urbanisés révèlent plus que d'autres.

À la veille de la réforme, les provinces qui devaient former le Nord et le Pas-de-Calais sont richement pourvues en juridictions, dont la plupart ressortissent au conseil provincial d'Artois (Arras) ou au parlement de Flandre (Douai). En Artois, sous l'autorité du Conseil provincial, la justice ordinaire du prince est rendue par la gouvernance d'Arras et les bailliages d'Avesnes-le-Comte, Hesdin, Saint-Omer, Bapaume, Lens et Airc ; des bailliages secondaires, des châtellenies ou prévôtés, secondés par une justice seigneuriale ou municipale active, complètent l'encadrement ${ }^{12}$.

(9) Dans les villes de plus de 50000 habitants, le nombre des juges pouvait être porté à six de manière à diviser le tribunal en deux chambres (décret du 16 août 1790, titre IV, art. 2). Une organisation spécifique fut établie à Paris par le décret du 25 août 1790 . voir Serge ABERDAM et alii, Voter, élire pendant la Révolution française. 1789-1799, Paris, CTHS, 1999, p. 167 et 171. Précisons aussi que, dans l'attente de l'installation des tribunaux criminels, un accusateur public fut adjoint au personnel ordinaire des tribunaux de district.

(10) Sur les enjeux de la réorganisation judiciaire : Marie-Vic Ozouf-Marignile, La formation des départements. La reprósentation du lerritoire français à la fin du 18 siècle, Paris, éd. EHESS, 1989, p. 220-226.

(11) Pour la liste des sièges de tribunaux, voir les Archives parlementaires, t. XVIII, p. 239-244.

(12) Philippe Suluk, Le Conseil provincial d'Artois (1640-1790). Une cour provinciale à la recherche de sa souveraineté, Arras, Mémoires de la commission départementale des monuments historiques du Pasde-Calais, t. XVIII-2, 1982, p. 466-507. 
Dans le Calaisis, le Boulonnais et la région de Montreuil, qui dépendent de l'intendance d'Amiens et ressortissent du parlement de Paris, la juridiction royale de Calais, la sénéchaussée du Boulonnais et le bailliage de Montreuil forment l'essentiel de l'armature judiciaire royale ordinaire's. Quant au ressort du parlement de Flandre, il regroupe un dense maillage de justices royales, seigneuriales et municipales du sein duquel émergent le présidial de Flandre (Bailleul), la gouvernance de Lille et les bailliages d'Avesnes et du Quesnoy.

$\grave{A}$ cette solide et ancienne armature correspond une population judiciaire dont l'importance transparaît de la lecture des almanachs provinciaux. Sans même évoquer le Boulonnais, le Calaisis et la majeure partie du Hainaut, l'espace septentrional rassemble plus de 150 juges, 500 avocats, 200 procureurs, 100 huissiers et sergents et 40 greffiers. Et ces chiffres ne sont que des estimations basses, qui n'offrent qu'un simple ordre de grandeur ; pour nombre de villes, les almanachs restent évasifs : ils ne mentionnent pas les avocats de Lens, n'évoquent pas les probables commis-greffiers d'Aire ou de Bailleul, tandis qu'il est bien difficile d'extraire des corps municipaux le personnel qui vit avant tout de la justice échevinale. Aux juridictions les plus importantes, d'ailleurs, l'on pourrait en ajouter bien d'autres. Ainsi, dans la capitale de l'Artois, le personnel judiciaire ne relève pas exclusivement du conseil souverain et de la gouvernance, puisque la justice y est également rendue par l'échevinage, l'élection provinciale, le siège prévôtal de la maréchaussée, la maîtrise des eaux et forêts, la prévôté de l'évêché, la justice temporelle du chapitre et le siège abbatial de SaintVaast $^{14}$; royales, municipales ou seigneuriales, ces diverses juridictions offrent du travail aux avocats et procureurs de la ville, mais entretiennent aussi leurs propres greffiers et sergents, tandis que leurs juges sont tantôt des officiers spécifiques - par exemple dans l'élection -, tantôt des avocats qui trouvent dans ces charges un supplément d'activité et de revenu. Les chiffres recueillis permettent cependant de mesurer l'ampleur du décalage annoncé entre l'ancienne et la nouvelle société judiciaire ; comment, en effet, autant d'hommes pourraient trouver place dans une structure allégée, simplifiée et géographiquement redistribuée ? À partir des exemples septentrionaux, trois situations différentes peuvent être isolées.

(13) Voir : Charles ENGRAND, "Réseau urbain et distribution des tribunaux royaux ordinaires dans la généralité d'Amiens (XVII-XVIII' siècle) ", Revue du Nord, $n^{\circ}$ 335-336, 2000), p. 257-269; Daniel Nordman, Marie-Vic Ozouf-Marignifr, Atlas de la Révolution française, 1. IV, Le territoire (1). Réalités et représentations, Paris, éd. EHESS, 1989, p. 80 ; Ghislaine Blllart, Pjerre Bougiard, Catherine Rollet, Paroisses et communes de France. Pas-de-Calais, Paris, éd. unjversitaires, 2 vol., 1975, passim.

(14) Almanach histonique et géographique d'Artois. Pour l'an de grâce 1789, Arras, veuve de Michel Nicolas, s.d, p. 77-85 (AD Pas-de-Calais, A 1101). 
Tableau 1.

Le personnel judiciaire de quelques villes des ressorts du Conseil d'Artois et du Parlement de Flandre ${ }^{15}$

\begin{tabular}{|c|c|c|c|c|c|c|}
\hline & Juges $^{16}$ & Avocats & Procureurs & $\begin{array}{l}\text { Huissiers } \\
\text { ou sergents }\end{array}$ & \begin{tabular}{|l|} 
Greffiers \\
et commis
\end{tabular} & $\begin{array}{l}\text { Tribunal } \\
\text { de district }\end{array}$ \\
\hline \multicolumn{7}{|c|}{ Ressort du Conseil d'Artois } \\
\hline Aire & 4 & 25 & 5 & 4 & 1 & \\
\hline $\begin{array}{l}\text { Arras: } \\
\text { Conseil d'Artois }\end{array}$ & $\begin{array}{l}26 \\
5\end{array}$ & 93 & 50 & $\begin{array}{l}9 \\
5\end{array}$ & $\begin{array}{l}5 \\
3\end{array}$ & Oui \\
\hline $\begin{array}{l}\text { Gouvernance } \\
\text { Bapaume }\end{array}$ & $\frac{5}{7}$ & 7 & 7 & \begin{tabular}{|l}
5 \\
5
\end{tabular} & \begin{tabular}{|l}
3 \\
3
\end{tabular} & Oui \\
\hline Béthune & 3 & 7 & 7 & 6 & 1 & Oui \\
\hline Dunkerque & & 31 & 5 & & & \\
\hline Hesdin & 10 & 20 & 6 & 4 & 1 & Oui \\
\hline Lens & 3 & $-\ldots$ & 4 & 5 & 1 & \\
\hline Saint-Omer & 10 & 23 & 8 & 6 & 2 & Oui \\
\hline Saint-Pol & 5 & 15 & 12 & 7 & 2 & Oui \\
\hline Total & & 233 & & & & \\
\hline \multicolumn{7}{|c|}{ Ressort du Parlement de Flandre } \\
\hline Bailleul & 13 & 37 & 12 & 6 & 1 & Oui \\
\hline Bavay & 2 & 8 & 4 & 4 & 1 & \\
\hline Bergues & & 39 & 4 & & & Oui \\
\hline Bouchain & 4 & $\begin{array}{l}2 \text { avocats } \\
\text { procure }\end{array}$ & $\begin{array}{l}+4 \text { avocats- } \\
\text { urs }\end{array}$ & 4 & 2 & \\
\hline Condé & 3 & 4 & 2 & 2 & 2 & \\
\hline $\begin{array}{l}\text { Douai: } \\
\text { Parlement } \\
\text { gouvernance }\end{array}$ & $\begin{array}{l}37 \\
9\end{array}$ & 91 & $\begin{array}{l}19 \\
6\end{array}$ & $\begin{array}{l}7 \\
7\end{array}$ & $\begin{array}{l}6 \\
2\end{array}$ & Oui \\
\hline Hazebrouck & & 13 & 3 & & & \\
\hline Le Quesnoy & 10 & 11 & 5 & 9 & 2 & Oui \\
\hline Lille & 10 & 44 & 41 & 12 & 4 & Oui \\
\hline Maubeuge & 2 & 24 & 6 & 8 & 1 & \\
\hline
\end{tabular}

C'est dans les villes judiciaires dépourvues d'un tribunal de district que la situation des juristes parait la plus délicate. En ces cas, la ville n'est pas toujours administrativement déclassée, tels Bergues et Hazcbrouck qui, tout en dépendant du tribunal d'une ville proche (Dunkerque et Bailleul),

(15) Estimation d'après l'Almanach historique et géographique d'Antois. Pour l'an de grace 1789, op. cit., s.d. (AD Pats-de-Calais, A 1101) et le Calendrier général du gouvemement de la Flandre, du Hainaut et du Cambrésis pour l'année 1789, Lille, Jacquez, s.d. (AD Pas-de-Calais, A 1103).

(16) Sans les honoraires, et sans les charges non occupées. 
sont chef-lieu de district et gardent le siège de l'administration. Reste que, pour le personnel judiciaire, la transition n'est guère aisée à gérer. Elle l'est moins encore dans les villes qui perdent également leurs fonctions administratives, telles Aire, Cassel, Lens, Condé, Bouchain, Bavay ou Maubeuge. C'est à Aire ( 8885 habitants $)^{17}$, sans doute, que l'impact social de la nouvelle carte judiciaire est le plus dur ; cette ville, qui a en vain sollicité le siège du département, n'obtient pas même un chef-lieu de district et voit disparaitre son bailliage principal, sa justice échevinale et sa justice temporelle du chapitre ; aux quatre juges royaux privés d'office, aux huissiers et sergents désormais sans charge, s'ajoutent vingt-cinq avocats et cinq procureurs privés d'emploi. La réforme, pour ceux qui souhaitent continuer d'exercer, inaugure une difficile période d'adaptation professionnelle : les avocats et juges peuvent se faire élire aux rares places de juge de district, les avocats continuer d'exercer, mais ailleurs, tout comme les procureurs et sergents ; il y a encore les emplois de juge de paix, ou les places permanentes dans les administrations nouvelles, particulièrement au district et au département... Ainsi, à l'issue des premières élections aux administrations de district, ce sont 33 juristes qui accèdent aux fonctions de président ou de membre du directoire des 16 districts du Nord et du Pas-de-Calais ${ }^{16}$. Pour les hommes issus de villes judiciairement déclassées, il ne faut pourtant exagérer ni l'importance des opportunités de la réorganisation administrative, ni la facilité des reclassements dans la justice nouvelle. Les procureurs de Cassel ne se plaignent-ils pas, en janvier 1791, de ne pouvoir exercer devant le tribunal de district de Bailleul, qui n'accepte que les anciens procureurs du présidial de la ville ? La loi des 29 janvier-20 mars 1791, d'ailleurs, ne va guère améliorer leur situation, puisqu'elle exige la résidence dans la ville du siège judiciaire. Les huissiers ne sont parfois pas mieux lotis ; ainsi, en dépit des textes, les juges élus de Valenciennes proposent au Comité de constitution de limiter l'accès aux fonctions d'huissier et de sergent devant leur juridiction par l'établissement d'un cautionnement (décembre 1790), dans le but avoué d'éloigner les professionnels autrefois domiciliés hors de leur commune ${ }^{19}$.

Dans les villes pourvues d'un tribunal de district, la situation est plus contrastée. Certes, l'abolition de la vénalité prive les magistrats de leurs charges, la liberté de la défense désorganise les professionnels du barreau, tandis que le remplacement des procureurs par des avoués pouvant exercer

(17) D'après l'enquête menée par le département en 1790. Léon-Noël BLrTHE, Pierre Bouciard, Danielle CANLer, Jean-Pierte Jessenne, Jean-Michel DeCELle, Villes et villages du Pas-de-Calais en 1790. Arras, Mémoires de la commission départementale d'histoire et d'archéologie du Pas-de-Calais, t. XXVIII, 1992, district de Saint-Omer.

(18) Hervé LEUWERS, “ 1790 : la formation du personnel des administrations de district. Lexemple du Nord et du Pas-de-Calais ", Revue du Nord, n 332, 1999, p. 745-773.

(19) AN, D IV 45, dossier Nord 1303, pièce 2 et dossier Nord 1302, pièce 12. 
défense et postulation transforme totalement les usages. Pour autant, les juges, greffiers et huissiers nouveaux, nous le verrons, sont prioritairement désignés parmi les anciens professionnels du lieu, tandis qu'anciens avocats et procureurs tentent un temps, comme à Bailleul, de s'assurer un quasi monopole de l'intervention devant la juridiction du district ${ }^{20}$. La prétention cependant, ne peut durer et, finalement, c'est dans des villes secondaires comme Bapaume, Béthune ou Saint-Pol, là où la nouvelle organisation judiciaire annonce un accroissement de l'activité judiciaire, là où le personnel judiciaire d'Ancien Régime est peu nombreux, que les vrais gagnants de la réforme peuvent s'observer.

Des seize villes qui obtiennent un tribunal de district, les deux qui perdent le plus sont les anciennes capitales judiciaires d'Artois et de Flandre ; à Arras et Douai, le tribunal nouveau ne peut offrir d'emploi à un personnel judiciaire inévitablement pléthorique ! À compter les seuls officiers des cours souveraines et gouvernances d'Arras et de Douai, ce sont 77 juges du siège ou du parquet qui perdent leur office alors que 10 places de juges de district sont proposées à l'élection; et que dire des 184 avocats, des 75 procureurs et des huissiers, sergents, greffiers ou commis qui ne peuvent tous espérer trouver de l'emploi dans un tribunal aux compétences et au ressort amoindris, dans les justices de paix ou les nouvelles administrations? Ici, plus qu'ailleurs, la remise en cause des statuts professionnels anciens, les bouleversements dans la manière d'exercer la justice entretiennent l'incertitude et obligent les gens de justice désirant poursuivre leurs activités à projeter un reclassement dans l'ordre nouveau.

\section{L'événement vécu : ampleur et faux-semblants d'un renouveau}

À observer les almanachs ou les sources judiciaires, l'ampleur sociale des changements apparaît d'abord pour les juges; elle est parfois moins évidente pour les avocats, les procureurs, voire les greffiers et les huissiers, particulièrement dans les sièges des tribunaux de district, comme si l'incertitude face aux réformes invitait au statu quo, comme si beaucoup, à défaut de préserver leur statut, pensaient conserver leurs fonctions, même sous un titre nouveau. Lesprit de continuité touche jusqu'à certains officiers qui accèdent aux places de juge, et dure au moins jusqu'à l'ouverture de ces fonctions à tout citoyen sans condition de compétences (19 octobre 1792) et au remplacement des avoués par d'informels « fondés de pouvoirs " ( 3 brumaire an II-24 octobre 1793). C'est qu'un réel décalage existe alors entre le discours de rupture qui accompagne les réformes 
constituantes et la manière dont les transformations de la famille judiciaire sont perçues par les juristes ou par les électeurs.

Pour les magistrats d'Ancien Régime, la réduction drastique du nombre des juges et leur désignation par les assemblées électorales forment autant de filtres qui ne permettent qu'à une petite part d'entre eux de poursuivre leur fonction ; cela est d'autant plus vrai que le décret des 16-24 août 1790, complété par celui des 25 août-2 septembre de la même année $^{21}$, précise que les juges de district et leurs suppléants doivent remplir les conditions du cens, être âgés de trente ans accomplis et avoir exercé pendant cinq ans les fonctions de juge... ou d'homme de $\operatorname{loi}^{22}$. Ces conditions d'éligibilité, formulées en de mêmes termes pour le « commissaire du roi », représentant de l'État nommé à vie par le monarque, offrent aux avocats une possible promotion professionnelle sans précédent. Et dans l'espace septentrional, comme dans les autres départements pour lesquels nous disposons de données chiffrées ${ }^{23}$, l'ampleur du changement alors provoqué est telle que moins du quart des anciens juges royaux intègre les nouveaux tribunaux.

Tableau 2.

Les juges de district élus du Nord et du Pas-de-Calais (1790)

\begin{tabular}{|l|l|l|l|l|l|l|}
\hline & \multicolumn{2}{|c|}{ Nord } & \multicolumn{2}{c|}{ Pas-de-Calais } & \multicolumn{2}{c|}{ Total } \\
\hline avocats & 12 & $29,3 \%$ & 16 & $40 \%$ & 28 & $34,6 \%$ \\
\hline avocats-notaires & --- & --- & 1 & $2,5 \%$ & 1 & $1,2 \%$ \\
\hline avocats-juges & 11 & $26,8 \%$ & 8 & $20 \%$ & 19 & $23,5 \%$ \\
\hline avocats-cultivateurs & 1 & $2,4 \%$ & -- & -- & 1 & $1,2 \%$ \\
\hline juges & 10 & $24,4 \%$ & 13 & $32,5 \%$ & 23 & $28,4 \%$ \\
\hline fonctions inconnues & 7 & $17,1 \%$ & 2 & $5 \%$ & 9 & $11,1 \%$ \\
\hline Total & 41 & $100 \%$ & 40 & $100 \%$ & 81 & $100 \%$ \\
\hline
\end{tabular}

(21) Décrets reproduits dans les Archives parlementaires, t. XVIII, p. 104-110 et 492-493.

(22) Cette condition d'excrcice n'est cependant pas exigée des protestants qui peuvent être élus s'ils ont été membres des municipalités, ou s’ils sont gradués en droit (décret des 25 août-2 septembre 1790, art. 6) ; le même article précise que l'Assemblée n'entend " rien préjuger par rapport aux juifs sur l'état desquels elle s'est réservée de prononcer ". Le même décret précise encore, dans ses articles 5 et 8 , que l'état ecclésiastique est incompatible avec les fonctions de juge, et que, pour la prochaine élection, les administrateurs ayant accepté de siéger dans les directoires, ainsi que les procureurs syndics et procureurs généraux syndics sont inéligibles.

(23) Voir notamment : Henry Jean-Louis DEBAUVE, La justice révolutionnaire dans le Morbihan. Essai sur l'organisation judiciaire du Morbihan de 1790 à 1795, Paris, chez l'auteur, 1965, p. 57-81 ; Jean-Claude ATruel, La justice, la nation, Versailles sous la Révolution, 1789-1792. La mise en place des tribunaux de district en Suine-et-Oise, Montgeron, Desbouis Grésil, 1989, p. 470-484; Elisabeth DANDINE, "Les élections judiciaires en Haute-Garonne (1790-an IV) ", dans Jacques KRYNEN, dir., L'élection des juges. Étude historique française et contemporaine, Paris, PUF, 1999, p. 77-93 ; Jean-Paul RotHot, « De l'officier au juge nommé : itinéraires de gens de justice vosgiens (1750-1800) ", Annales de l'Est, 1998, $\mathbf{n}^{\circ} 2$, p. $421-422$. 
Une approche d'ensemble conduit à insister sur le profond renouvellement des juges, et la faible place qu'y tiennent les anciens officiers royaux : seuls dix d'entre eux dans le Nord et treize dans le Pas-de-Calais occupent la fonction de juge du siège ! Même si les places de commissaires reviennent essentiellement à d'anciens membres du parquet, les exclus - consentants ou non - sont nombreux. Comme dans le Morbihan, la Seine-et-Oise ou la Haute-Garonne, ce sont avant tout des avocats qui obtiennent les suffrages publics. Leur présentation, cependant, rappelle la complexité de ce statut professionnel sous l'Ancien Régime, où l'excrcice simultané de plusieurs fonctions n'est pas rare. Reste qu'à côté d'un notaire-avocat et d'un cultivateur-avocat, probablement guère présents au barreau, ce sont avant tout de vrais défenseurs qui entrent dans la carrière ; parmi eux, les électeurs ont valorisé les plus solides expériences professionnelles, qui transparaissent notamment dans ce grand nombre d'avocats ayant occupé une fonction de juge dans une juridiction seigneuriale ou un bailliage $^{24}$. À Saint-Omer, la figure de Deschamps de Pas est emblématique ; ancien avocat et conseiller du roi au bailliage de sa ville, il est élu administrateur de district, puis juge au tribunal de la même circonscription. Dans ses mémoires, il commence le récit de cette dernière élection en écrivant : « C'est ici qu'il faut appliquer le proverbe "à malheur quelque chose est bon" ", puis insiste sur sa nette préférence pour les fonctions judiciaires ${ }^{25}$. Pour comprendre ces premières élections, cependant, il faut rappeler que leurs résultats varient d'un district à l'autre et laissent apparaître des enjeux localement différenciés.

En de nombreux districts, comme Le Quesnoy (Nord), Calais et Hesdin (Pas-de-Calais), les électeurs ont partagé leur confiance entre avocats et magistrats. Dans d'autres, les avocats locaux l'ont clairement emporté, comme à Saint-Omer et Boulogne (Pas-de-Calais) où ils occupent quatre des cinq sièges, comme à Cambrai (Nord) où les cinq élus appartiennent au barreau local. Les sources ne livrent aucune certitude quant à l'interprétation à donner de ces choix. Il est possible qu'en certaines villes, une pré-campagne en faveur des avocats ait emporté l'adhésion des électeurs ; en Artois, cependant, l'Avis important d'un fermier des environs d'Arras sur le choix des juges qui doivent composer les tribunaux de district, très hostile aux magistrats de l'ancien conseil souverain, n'a pas dissuadé les électeurs de les désigner en prioritée ${ }^{2 t}$. Les choix peuvent également

(24) Un tableau comparatif concernant le Pas-de-Calais, la Seine-et-Oise et le Morbihan est présenté dans Hervé Leuwfrs, « 1790 et la naissance du juge-citoyen. Lexemple du Pas-de-Calais », dans Société et religion en France et aux Pays-Bas. $X V^{\prime}$-XIX' siècle. Mélanges en l'honneur d'Alain Lottin, Arras, APU, 2000, p. 602.

(25) Louis Auguste Deschamps de Pas, Ma vie publique, Saint-Omer, Société des Antiquaires de la Morinie, 2003, p. 33.

(26) AD Pas-de-Calais, collection Barbier A 1285, Avis important d'un fermier des environs d'Arras, sur le choix des juges qui doivent composer les tribunaux de district, Lille, imp. L. Danel, 1790, 3 p. 
s'expliquer par des tensions locales, parfois perceptibles dès la rédaction des cahiers de doléances, comme à Saint-Omer, où le cahier du Tiers état propose la suppression de la vénalité et le choix des juges par les « justiciables parmi les avocats qui auront exercé leur profession pendant au moins dix ans ${ }^{27}$. Plus efficaces, sans doute, ont été les débats qui, au cours des assemblées électorales, ont invité les électeurs à se déterminer en examinant le patriotisme des candidats, comme à Hesdin (Pas-de-Calais), où cette revendication est mentionnée au procès-verbal, qui précise que les juges « devroient être non seulement instruits et vertueux, mais encore amis de la constitution ${ }^{28}$.

Mais c'est dans le résultat même des élections que l'enjeu patriotique du scrutin apparaît le plus nettement. Si, dans les départements septentrionaux, la place des Constituants parmi les élus reste bien plus modeste qu'à Paris où quatorze des trente juges de district appartiennent à l'Assemblée $e^{29}$, elle n'en est pas moins révélatrice à la fois de stratégies de renforcement du prestige des tribunaux nouveaux et de valorisation de parcours patriotes ; ainsi, dans le Pas-de-Calais, trois députés et un suppléant du Tiers aux États généraux sont élus juges à Arras, Boulogne et Calais, tandis que, dans le Nord, le constituant Gossuin est élu au Quesnoy $^{30}$. Si l'on prend également en compte les élus municipaux, des districts et des départements au moment des élections de 1790 , ce sont cette fois quinze des quarante juges du Pas-de-Calais qui disposent d'un mandat électif au moment de leur désignation, ce chiffre étant d'au moins neuf sur quarante dans le Nord ${ }^{31}$. Limportance de ces chiffres, sans doute également liée à la nature du système électoral dans lequel l'absence fréquente de candidatures conduit les électeurs à désigner des personnalités déjà en vue - par commodité ou pour valider une confiance accordée par l'accession à l'assemblée électoralc -, n'en demcure pas moins un signe fort de la dimension politique des nouvelles fonctions judiciaires. D'ailleurs, lorsque des contestations électorales existent, qu'elles portent sur la régularité des élections ou l'éligibilité des promus, elles s'accompa-

(27) Art. 33. Voir Jean-Pierte JESSENNE et Dominique RosselLE, op. cit., p. 165.

(28) AD Pas-de-Calais, 2L6/148, en date du 13 octobre 1790.

(29) A. Dovarche, Les tribunalux civils de Paris pendant la Révolution (1791-1800), Paris, Léopold Cerf-Noblet-Quantin, t. I, 1905, p. 4-5.

(30) J.F.J.H. Thellier à Arras, N. Latteux et B. Gros à Boulogne, C.B. Francoville à Calais.

(31) Dans le Pas-de-Calais, outre les trois Constituants et le suppléant aux États généraux, on compte parmi les juges de district élus en 1790 neuf élus municipaux, un administratcur de district et un de département. Dans le nord, on dénombre un Constituant, un administrateur du département, deux ałdministrateurs et un secrétaire de district ainsi que quatre élus municipaux (à la liste donnée dans Hervé LeUwERs, «Élire les juges. Lexemple des juges des tribunaux de district du Nord et du Pas-de-Calais. 17901792 », dans Robert ChaGNy, dir., La Révolution française. Idéuur, singularités, influences, Grenoble, PUG, 2002, p. 315, jajouterai deux juges de Cambrai : Rodriguez, élu à Cambrai, et Doutart, élu au CateauCambrésis. Voir AN, D IV 46, dossier 1333. pièce 12, 8 octobre 1790 et AD Nord, L 6898, procès-verbal d'élection des juges de Cambrai, octobre 1790). 
gnent souvent d'allusions au positionnement politique des intéressés ; ainsi, dans une lettre dénonçant les liens de quatre des juges de Cambrai avec l'Église, Trocmé, administrateur du directoire du département, s'étonne que le seul élu dont la désignation soit remise en cause n'est autre que l'avocat François Delsaux, qu'il qualifie de «bon citoyen et de veritable ami de la constitution $»^{32}$.

Mais ce patriotisme, parfois explicitement recherché, n'est pas le moteur de toutes les élections de juge ; ainsi, les districts d'Arras et de Douai, dont les chefs-lieux ont abrité les cours souveraines d'Artois et de Flandre, échappent au schéma habituel et trahissent une perception différente des enjeux de la réorganisation judiciaire, sans doute présente simultanément chez les gens de justice et chez les électeurs de ces circonscriptions. Ne remarque-t-on pas, en effet, qu'y ont prioritairement été désignés d'anciens magistrats royaux, parfois membres de l'ancienne cour souveraine ? C'est la recherche du prestige et l'affirmation d'une continuité avec la cour perdue qui transparaît à Arras, où sont élus cinq magistrats du Conseil d'Artois ${ }^{33}$. À Douai, comme dans les villes de Rennes ou de Toulouse ${ }^{34}$, les électcurs se montrent moins enclins à désigner d'anciens parlementaires même si, en l'occurrence, le quatrième juge est Dubois, ancien conseiller à la cour ; mais les trois postes attribués à des officiers de la gouvernance permettent de confier le tribunal à une majorité de juges d'Ancien Régime et, ici aussi, de revendiquer une continuité avec la justice disparue ${ }^{35}$. La même recherche d'un prestige, probablement liće à une valorisation de compétences présumées, se retrouve dans des districts proches des anciennes capitales judiciaires. Dans le Pas-de-Calais, à la manière de ce qui s'est produit en Bretagne, les électeurs accordent ainsi leur confiance à des juges ou à des avocats de l'ancien Conseil provincial à Béthune ( 3 juges sur 5 ), à Saint-Pol ( 2 juges sur 5 ) et à Bapaume ( 3 juges sur 5$)^{36}$. Dans le Nord, les choses sont moins nettes et l'influence de la magistrature du parlement ne dépasse pas le district de Douai, tandis que

(32) AN, D IV 46, dossier 1333, pièce 12,8 octobre 1790.

(33) J.F.J.H. Thellier, M.F.M. Thiébault, J.R.T.B.J. Lefebvre, P.F.J.M. Dourlens et P.I.J. Lecocq. Voir Philippe SUEUR, op. cit., t. XVIII, v. II, 1982, p. 860-861 et [PLoUvaIN], Notes historiques relatives aux offices el aux officiers du Conseil provincial d'Anois, Dosuai, imp. Wagrez aîné, 1823, p. 54, 73, 75, 76, 97, 98.

(34) Marcel Morabito, dir., La Révolution et les juristes a Rennes, Paris, Economica, 1989, p. 6-63 ; Elisabeth DANDINL, op. cit., p. 79-82.

(35) H.I.M.J. Duhamel, lieutenant général civil et criminel à la gouvernance, J.F. Leroux, conseilles à la gouvernance, A.F.G. Wagon, conseiller à la gouvernance, P.H. Dubois, conseiller au Parlement et M.J. Dupont, avocat et avocat fiscal de la cour féodale et temporelle du chapitre de SaintAmé de Douai. AD Nord, L, 12473, procès-verbal de l'asscmblée électorale du district de Douai (octobre 1790), et vérification des pièces attestant de l'âge et des capacités des juges élus (12 novembre 1790 ).

(36) À Béthunc, les avocats T.C. Dewez, H.B.J. Lobez et C.A.J. Dourlens ; à Saint-Pol, M.P.E. Herman, substitut de l'avocat général du Conseil d'artois, et l'avocat E.F.J. Petit ; à Bapaume : P.A.H. Lesage (avocat au Conscil d'Artois et avocat du roi à la gouvernance d'Arras), L.J. Carion (avocat au Conseil d'Artois et président en l'élection d'Arras) et L.J. Audefroy, conseiller au Conseil d'Artois. 
celle de son barreau apparaît bien faible, puisque seul Lille nomme pour juge l'un de ses membres. Dans cette dernière ville, cependant, en vain candidate au chef-lieu du département, les électeurs ont nommé juges, outre un avocat du parlement et un avocat lillois, trois magistrats de la gouvernance et un conseiller pensionnaire de la ville, également ancien juge royal ${ }^{37}$; par leurs choix, comme à Arras ou à Douai, ils se sont également montrés sensibles au prestige de leur nouveau tribunal.

L'attitude des électeurs renvoie à une perception de la réforme moins radicale qu'on peut l'imaginer. Bien des indices signalent que l'obtention d'un tribunal de district peut être perçu, non seulement comme l'occasion de préserver une activité judiciaire vitale, mais aussi comme la reconnaissance de l'excellence des personnels locaux, et des compétences, du savoir faire et de la qualité du service judiciaire rendu sous l'Ancien Régime. Dans la correspondance échangée avec le Comité de constitution, il n'est pas rare d'entendre évoquer la prétention des juges nouveaux à prolonger les travaux de prédécesseurs dont ils se disent les héritiers, tels ces juges de Bailleul qui assurent que leur tribunal « représente le bailliage rö̈al et siege présidal de Flandres supprimé » $(1791)^{38}$. La continuité des fonctions s'imagine plus encore au niveau individuel; qu'on se rappelle le beau portrait du juge Pierre Lecocq par Dominique Doncre, aujourd'hui conservé au Musée de Vizille. Aux côtés de sa femme et de ses enfants, le juge du district d'Arras porte l'habit noir de sa fonction, médaille en sautoir et une main posée sur son chapeau ; mais en arrière plan, sur le côté droit du tableau, un petit génie de la liberté - bonnet rouge à la main - n'a pas achevé de tirer un rideau devant la robe rouge et le bonnet de son ancien office au conseil d'Artois. Dans cette représentation familiale, le passé n'est ni occulté, ni réprouvé ; c'est au contraire la transition de l'office de conseiller à la fonction de juge élu et la permanence du prestige social qui se donnent à lire.

Cette continuité des fonctions a été plus encore ressentie par les commissaires du roi, dont la nomination reste à la disposition du roi, ou par les greffiers. Dans le Nord et le Pas-de-Calais, ce sont généralement les anciens procureurs du roi aux bailliages qui obtiennent les nouvelles places de commissaires du roi, comme à Douai, Arras ou Bapaume. Et lorsqu'il en est autrement, l'incident éclate, comme à Lille, où l'ancien procureur du roi à la gouvernance, Frémicourt, est écarté au profit de Pajot, secrétaire de l'intendance ; l'ancien officier s'en plaint en développant un raisonnement

(37) A.C.J. Lamblin, lieutenant particulier civil et criminel à la gouvernance, J.B.J. Dubrule, conseiller à la gouvernance, P.F.J. Danel, conseiller à la gouvernance, L.A.S. Demasur, conseiller pensionnaire de la ville et conseiller honoraire à la gouvernance. H.C.J.B. d'Haubersart, avocat au Parlement de Douai, L.J. Bara, avocat à Lille. AD Nord, L 4901, procès-verbal de l'ćlection des juges du district de Lille. Voir aussi M. PRÉvost, "Les élections judiciaires à Lille en 1790 ", Revue du Nord, 1958, p. 357-361.

(38) AN, D IV 45, dossier 1303, pièce 2 (janvier 1791). 
dans lequel le premier argument dénonce une blessure d'amour-propre : «La préférence donnée à un secrétaire d'intendant, est une vraie flétrissure. Je n'en ai mérité aucune ", écrit-il à l'Assemblée ; au Garde des Sceaux il affirme : « Cette exclusion particulière donne la plus vive atteinte à ma réputation qui n'a jamais été fletrie ${ }^{33}$; ce n'est qu'après - comme si cela n'était pas l'essentiel - qu'il dénonce l'infraction au décret sur l'organisation des tribunaux, assurant que Pajot n'a jamais exercé cinq années comme juge ou avocat. Pour Frémicourt, soutenu par les députés du Nord et la Société des amis de la constitution de Lille, la continuité du ministère public s'impose lorsque l'ancien officier a rempli au mieux ses missions ${ }^{40}$. C'est la même reconnaissance de compétences éprouvées, la même conviction que certains acteurs de l'ancienne justice gardent leur place et leur légitimité dans la nouvelle, qui explique la reconduction, par les juges, de nombreux greffiers des anciennes juridictions. Certes, les places ouvertes sont moins nombreuses que les anciennes; dans les villes qui demeurent centre judiciaire, cependant, c'est le plus souvent l'ancien greffier de juridiction qui obtient l'emploi, tel l'ancien greffier de la gouvernance de Douai, Dumortier, ou le « ci-devant greffier » du bailliage royal d'Avesnes, Bevière ${ }^{41}$. À Saint-Omer, le juge Deschamps de Pas relate que son confrère Duval, issu d'Aire, a tenté de faire désigner comme greffier du tribunal de district le procureur Dullu, issu de sa ville ; en réponse à sa proposition, Deschamps lui écrit : «Si j'avais à me prononcer en faveur de quelqu'un je me prononcerais infailliblement pour le Sr Dullu qui réunissait toutes les qualités possibles pour cela... mais qu'il me coûtait de déplacer quelqu'un lorsqu'on avait aucun reproche à lui faire, que l'ancien greffier devait mériter la préférence ». Allart, ancien greffier du bailliage, a alors été élu par trois voix contre deux, laissant transparaître les concurrences entre personnels judiciaires issus de Saint-Omer et d'Aire ${ }^{42}$. À n'en pas douter, nombre d'anciens greffiers ou commis ont également pu trouver de l'emploi auprès des juges de paix.

La même préservation de l'activité, à défaut de celle de l'état, se retrouve chez les auxiliaires de justice placés au service des justiciables, chez ceux au moins qui exercent réellement la fonction attachée à leur titre. Dans l'attente des mesures sur les avocats et procureurs, les anciens corps professionnels demeurent inchangés, et les almanachs de 1790 publient des listes très proches de celles de l'année précédente. Dans le ressort de l'ancien Conseil d'Artois, les barreaux de Bapaume, Béthune, Bourbourg, Dunkerque, Hesdin, Saint-Omer et Saint-Pol restent stables,

(39) AN, D IV 45, dossicr 1301, pièce 5,29 octubre 1790 et pièce 7, 25 octobre 1790 .

(40) AN, D IV 45, dossier 1301, notamment pièces 5-16.

(41) BM Douai, Ms 1006, p. 179 (pour Dumorticr) ; AD Nord, L 12163, commission des juges d'Avesnes (pour Bevière)

(42) Louis Auguste Deschamps de PAs, op. cit., p. 35. 
tandis que celui d'Arras passe de 93 à 92 inscrits, et celui d'Aire de 25 à 24 . La même remarque s'applique aux procureurs. Lorsque les almanachs existent, comme à Lyon, Limoges ou Paris, la préservation de ces listes de professionnels est encore attestée pour $1791^{43}$. C'est que, s'ils n'ont pas intégré l'administration du district ou du département, ou un siège de juge de paix ou de district, leur activité antérieure peut se poursuivre par d'autres voies ; ainsi, au lendemain de la suppression de l'état et des ordres d'avocat, Lebeau, ancien défenseur d'Avesnes peut assurer exercer « la profession d'homme de loi ${ }^{44}$.

Il n'est pas douteux, en effet, que même si la préservation de listes de professionnels relève en partie de l'habitude ou du formalisme, l'ordre nouveau leur laisse un espace de travail qui demeure large ${ }^{45}$. Au pénal, dès la loi des 8 octobre-3 novembre 1789 , la redéfinition des droits de la défense ouvre aux " hommes de loi » de nouvelles carrières ${ }^{46}$. Au civil, les procureurs - puis les avoués - restent des auxiliaires très sollicités, tandis que les défenseurs, même concurrencés, conservent un rôle d'autant plus essentiel que le maintien de la procédure de 1667 et du pluralisme juridique d'Ancien Régime préserve la technicité de la justice du district. Lon peut ajouter que l'immixtion rapide et fréquente des hommes de loi dans les procédures de conciliation et d'arbitrage, pourtant destinées à repousser le moment de l'accès à la justice proprement dite, leur offre également de nouvelles occupations ; plutôt que de choisir pour arbitre un parent, voisin ou ami, on préfère demander à un juriste de trancher, quand il ne s'agit pas de faire siéger des hommes de loi dans un tribunal de famille ${ }^{47}$. De la même manière, on n'hésite pas à solliciter l'appui d'un procureur ou avoué, ou d'un homme de loi pour des procédures conciliatoires ; bien décidé à résister à cet envahissement, le président du bureau de paix de Bailleul le dénonce au Comité de constitution en janvier 1791, s'inquiétant de ces procureurs qui prétendent représenter ou accompagner la personne appelée à comparaître : «Vous pouvez aisément concevoir, écrit-il, que

(43) Calendrier de la ville de Limoges /... /. Limoges, L. Barbou, s.d., année 1791 ; Almanach de la ville de Lyon et du département de Rhône et Loire, pour l'année 1791, Lyon, A. de la Roche, 1761 ; LAlmanach royal, année commune M.DCC.XCI, Paris, V"d d'Houry, s.d.

(44) Souligné par moi. AN, D IV 46, dossier 1333, pièce 2 (13 octobre 1790).

(45) Jean-Louis HALPERIN, " Droit de défense et droits des défenseurs en France de 1789 à 1914 ", dans L'assistance dans la résolution des conflits, t. LXIV des recueils de la société Jean Bodin, Bruxelles, De Boeck Université, 1997, notamment p. 99-110.

(46) Nicolas DeRAsse. La défense dans le procès criminel sous la Révolution et le premier empire (1789-1810) : les mutations d'une fonction et d'une procédure, thèse doctorat, Lille II (dir. J.P. Royer), 1998, t. I, p. 48-57. Jean-Pierre RoYf.R, “ Parole d'avocat... Remarques sur la plaidoiric pénale, de la fin de l’ancien régime à la Révolution ", Droits, $n^{\circ} 17,1993$, p. 99-112.

(47) Jean-Louis HALPÉRIN, "Tribunaux de famille. Les juristes ou comment s'en débarrasser ? ", dans La famille, la loi, l'État de la Révolution au Code civil, Paris, Ed. du Centre Georges Pompidou, 1989, p. 292-304 ; Véronique Demars-Sion, “ La justice révolutionnaire en accusation : les griefs d'un nommé Saugeon contre les tribunaux de famille lillois $»$, Les épisodiques, $n^{\circ} 6,1993$, p. $21-37$. 
leur interest n'est pas que les parties se concilient ${ }^{48}$ ! Deux mois plus tard, leur intervention est cette fois explicitement interdite par la loi (6-27 mars $1791)^{43}$.

Les exclus de la nouvelle justice, bien sûr, sont nombreux et la rupture est d'une ampleur inconnue jusque là ; pour autant, tout invite à une lecture contrastée des bouleversements professionnels des débuts de la Révolution, et à une mise en évidence du décalage entre l'ampleur de la réforme annoncée et la relative continuité du personnel chargé de la mettre en œuvre. Mais surtout, alors qu'à l'Assemblée, sur les ruines du pluralisme judiciaire et de l'image du roi « fontaine de justice », s'élabore un discours de rupture, la réforme des structures judiciaires est diversement comprise dans le pays. Pour les uns, le bouleversement qui s'annonce doit être aussi profond que la réforme pénale et doit entraîner une mutation professionnelle et sociale d'ampleur ; mais pour d'autres, sans doute les plus nombreux, le service des anciens tribunaux n'est en rien contradictoire avec celui de la Nation, la réforme devant s'accompagner d'une préservation de l'activité du plus grand nombre possible des juristes d'autrefois. De fait, la refondation judiciaire ne fait alors que commencer et, pour les contemporains, ses dimensions les plus radicales ne se font pas encore sentir.

\section{L'aube d'une refondation}

Malgré les fréquents espoirs d'un reclassement dans l'ordre nouveau ou d'une préservation de certains héritages, les réformes constituantes transforment les activités de juge, d'avocat, de procureur, de greffier et d'huissier au-delà de ce que les contemporains ont pu penser aux débuts de la Révolution. Pour autant, ces transformations ne vont pas toujours dans le sens voulu par les Constituants... Qu'elles prolongent des changements à l'œuvre dès le XVIII' siècle, ou instaurent de nouvelles pratiques ou cultures professionnelles, elles ne font pas immédiatement sentir toute leur importance.

Dès 1789, une forte méfiance envers les gens de justice conduit à vouloir limiter leur nombre et leur rôle dans la société ; au sein d'une assemblée où les juristes sont pourtant surreprésentés ${ }^{s u}$, l'appel d'un Mirabeau à « contenir ou anéantir tous les subalternes suppôts de la justice, les huissiers, les sergents, les procureurs et les avocats " trouve un

(48) AN, D IV 45, Dossier 1303, pièce 3.

(49) Jean-Louis HalpÉRin, « Droit de défense et droits des défenseurs... *, op. cü., p. 103.

(50) Euna H. LEMAY, « Les révélations d'un dictionnaire : du nouveau sur la composition de l'assemblée nationale constituante (1789-1791) ", $A H R F, \mathrm{n}^{\circ} 284,1991$, p. 159-189. 
écho étrangement favorable ${ }^{\text {st }}$. Le recul de la chicane, pense-t-on, passe par un allègement des structures judiciaires formelles, une mise à l'écart de personnels soupçonnés de prolonger par intérêt les procédures... Avec la disparition des corps professionnels, la figure du juge de paix - particulièrement dans les campagnes ${ }^{52}$-, la désorganisation des facultés de droit et l'absence de mesures organisant la formation des futurs juristes, c'est une déprofessionnalisation relative qui s'annonces. ${ }^{53}$. Mais l'application de la réforme conduit aussi à favoriser certains changements qui, au contraire, favorisent une possible accélération des processus de professionnalisation à l'œuvre au XVIII' siècle! Ainsi, la réorganisation judiciaire provoque une diminution conséquente du nombre des juges, des huissiers et greffiers, et bientôt des avocats et avoués, qui assure une meilleure occupation professionnelle aux juristes en place. Elle s'accompagne, d'ailleurs, pour le juge, le greffier ou l'avoué, d'une interdiction de cumuler diverses fonctions ; le temps du procureur-notaire, de l'avocat-procureur ou de l'avocat-juge est bien terminé. Parallèlement, la suppression des ordres prive d'état nombre d'avocats en titre, qui n'ont jamais exercé, et assimile ainsi la fonction d'homme de loi - et plus encore de défenseur officieux - avec une activité réelle. En parlant du défenseur officieux, la distinction autrefois faite " entre le titre \& la profession d'avocat " perd toute signification ${ }^{54}$. Paradoxalement, le nom de "défenseur officieux », qui devait signaler l'ami ou le citoyen dévoué, en vient à désigner plus souvent un vrai professionnel que l'ancien mot " avocat »!

La réorganisation judiciaire, bien qu'assurant à nombre d'acteurs judiciaires la préservation de leur fonction ou un reclassement, permet aussi un profond renouvellement des hommes. Si les juges, nécessairement choisis parmi les juges, avocats ou professeurs de droit sont des juristes installés, il n'en va pas de même des nouveaux juges de paix, défenseurs officieux et avoués. Sans évoquer ici le cas des juges de paix, que le législateur veut placer en bordure du pouvoir judiciaire, rappelons qu'aux côtés d'anciens avocats, quelques nouveaux venus investissent la défense officieuse ; à Douai, ils sont cependant peu nombreux et n'apparaissent vraiment qu'à partir de 1792 et devant le tribunal criminel ${ }^{55}$. Louverture doit également toucher les avoués, l'accès au titre étant permis non seulement aux anciens juges ou avocats - peu séduits -, et aux anciens procureurs, mais

(51) Archives parlementaires, t. VIII, p. 395 (11 août 1789). Voir Jean-Louis HAL.PÉRIN, « Haro sur les hommes de loi ", Droits, $n^{\circ} 17,1993$, p. 55-65.

(52) Sur les juges de paix voir, notamment : Jacques-Guy Ptrtr, dir., Une justice de proximité : la justice de paix, 1790-1958, Paris, PUF, 2003, p. 35-64 (Serge Bianchi, Claude Coquard, Claudine DurandCoquard); Guillaume MrtaIRIF, Le monde des juges de paix de Paris, Paris, Loysel, 1994.

(53) Jean-Louis HALPÉRIN, " Haro... ",op. cit., p. 60.

(54) "Avocat ", dans DENISART, Collection de décisions nouvelles et de notions relatives à la jurisprudence, t. II. Paris, V“ Desaint, 1783, p. 708.

(55) Nicolas DeRASSE, op. cit., t. I, p. 148-151. 
aussi aux clercs de ces derniers lorsqu'ils disposent de plus de cinq ans d'ancienneté ; si les anciens professionnels tentent un moment d'cmpêcher la promotion de leurs anciens clercs, comme à Cambrai, ils doivent bientôt plier devant la loi ${ }^{\text {st }}$. Un vaste brassage commence, une culture nouvelle se met en place, tant chez les juges que chez les défenseurs officieux et les avoués ; d'horizons divers, ils se retrouvent désormais dans une occupation réelle et souvent exclusive de leurs fonctions.

Plus encore qu'au temps des Lumières, l'État est le premier acteur de ces transformations professionnelles ; par ses décisions législatives, il accentue le caractère national des groupes nouvellement définis. C'est l'État qui précise les compétences obligées du juge de district et de l'avoué, c'est lui encore qui, en privant le défenseur d'un « état » exclut de la défense officieuse tous ces avocats en titre, intéressés par l'honneur que confère leur titre. À la diversité professionnelle de l'Ancien Régime, où la nature et les prérogatives de ces différentes fonctions varient d'une province à l'autre, voire d'une juridiction à l'autre, succèdent des activités uniformément définies par la loi. Cette unité nouvelle, dans un premier temps, ne conduit aucunement à des rapprochements entre professionnels ; le nouveau monde des gens de loi, au contraire, se montre incapable de la moindre mobilisation commune. Pour autant, un pas essentiel a été franchi ; tandis qu'à la frontière du judiciaire il apparaît dans le « notaire public » qui succède aux figures multiples du tabellion et des notaires royaux, seigneuriaux ou apostoliques ${ }^{57}$, il touche aussi les juges, greffiers, huissiers, hommes de loi et avoués, dont le statut est désormais uniformément défini.

Une dernière rupture, cette fois sociale, est en gestation ${ }^{5 x}$. La suppression des offices, la disparition des privilèges et bientôt l'abolition de la noblesse héréditaire, entraînent l'effondrement de l'imaginaire social des gens de loi, et particulièrement des anciens magistrats et avocats, longtemps attachés à la « noblesse du droit ». D'origine romaine, mais réactivée au bas Moyen Âge, cette distinction est reconnue aux gradués des universités qui, par leurs origines ou leurs fonctions, n'appartiennent pas, de fait, au deuxième ordre ; vécue différemment d'une province à l'autre, plus concrètement en pays de droit écrit qu'en pays de coutume, elle assure à l'homme de loi un statut parfois accompagné de privilèges, comme l'exemption du droit de guet et garde, du logement des gens de guerre ou

(56) AN, D IV 45, dossier 1303, pièce 4 (22 février 1791).

(57) Sur la réforme du notariat, voir : Fred STEvens, La loi de ventôse contenant onganisation du notariat et sa genèse, Bruxelles, Bruylant, $2004 \mathrm{ct}$, pour un exemple régional, Sébasticn Jahan, Profession, parenté, identité sociale. Les notaires de Poitiers aux temps modernes (1515-1815), Toulouse, Presses universitaires du Mirail, 1999, p. 195-220.

(58) Lidée a été développée dans Hervé LeUwERs, « La robe révolutionnéc. Quand les gens de loi renoncèrent à la noblesse du droit (1780-1810) ", dans Jean-Pierre JESSENNE, dir., Vers un ordre bourgeois ? Révolution française et changement social, Rennes, PUR, 2007, p. 105-118. 
de la collecte des impôts ${ }^{59}$. Tout un ensemble de représentations sociales viennent conforter cette prétention : des références aux illustres prédécesseurs romains (sénateurs ou orateurs selon les professions), aux comparaisons entre l'utilité sociale du juge et celle du soldat, ou à la conviction d'un déclassement social récent... Et tandis que le juge et l'avocat rêvent de noblesse, quelques juristes tentent de préserver l'image sociale du procureur, assurant que son état est non dérogeant s'il exerce devant une cour $r^{\text {kt) }}$. Avec la Révolution, ces représentations sociales s'effacent ; et même l'invention de la noblesse impériale, qui récompense pourtant les principaux magistrats de l'Empire, ne pourra rétablir ces références si fréquentes au temps des Lumières. La noblesse cesse d'être une prétention des gens de loi.

Le Nord et le Pas-de-Calais ne sont qu'un exemple, parmi d'autres ; une observation plus large des phénomènes à l'ouvre en ces débuts de la Révolution permettra de compléter le tableau, de le nuancer, de l'agrémenter de nuances régionales... De ce premier cadre d'observation, on peut au moins apercevoir certaines implications sociales majeures de la réforme judiciaire ; les mettre en évidence, c'est insister sur leurs contradictions, sur les décálages entre les objectifs des députés et leurs réalisations concrètes, sur les potentialités longtemps insoupçonnées d'une rupture qui devait influencer durablement l'organisation de la famille judiciaire. Audelà d'une compréhension diverse des mesures de l'Assemblée et de ses objectifs par les gens de loi, c'est une recomposition sans précédent de la famille judiciaire qui s'opère : les juges élus, par leurs fonctions, leurs statuts ou leurs personnes, ne sont plus les officiers de l'Ancien Régime ; les statuts, les activités et la composition du groupe des hommes de loi et des avoués soulignent, de la même manière, l'ampleur de mutations, qui épargnent sans doute davantage le petit personnel des greffiers ou huissiers... La volonté de déjudiciariser la société, de faire reculer la chicane et l'influence sociale des gens de droit s'accompagne certes d'une ouverture sans précédent aux non juristes et d'une désorganisation de l'ancienne vie des corps professionnels, mais contribue aussi au resserrement géographique et fonctionnel, et à une diminution du nombre des anciens professionnels du droit, qui permettent implicitement la poursuite de certaines formes de professionnalisation. Facilité par l'ouverture des fonctions judiciaires, le rejet de la vénalité, de la noblesse et du privilège, le renouveau des juges et défenseurs conduit à un changement durable du position-

(59) Sur la revitalisation italienne et médiévale de cette noblesse, voir Patrick GıLl, La noblesse du droit, Paris, Honoré Champion, 2003. Voir aussi, Hervé LEUWERS, "La robe révolutionnéc... ", op. cit., p. $109-113$.

(60) Encyclopédie, ou dictionnaire raisonné des sciences, des arts et des métiers, Genève et Neufchatel, J.L. Pellet et Société typographique, t. 27, 1779, p. 503-504. J.N. GuYoT, Répertoire universel et raisonné de jurisprudence, Paris, Visse, t. XIII, 1784, p. 717. 
nement social de ces groupes et à leur abandon de toute référence à la "noblesse du droit». Pour la première fois, enfin, une définition nationale de tous les groupes de gens de loi, du juge à l'huissicr, est introduite par la loi. Cette réforme constituante, d'une ampleur professionnelle et sociale trop peu soulignée, est certes rapidement remise en cause par la réorganisation de l'élection des juges (1792), la suppression des avoués (1793), la Terreur puis les réformes judiciaires de l'an III et de l'an VIII ; mais bien que souvent transitoires, ses conséquences sur les cadres professionnels, sociaux et culturels de l'ancienne société judiciaire vont avoir une influence déterminante sur la formation de la société judiciaire contemporaine.

Hervé LEUWERS

Université Lille 3 / IRHiS

BP 60149

59653 Villeneuve-d'Ascq

herve.leuwers $@$ univ-lille3.fr 